\title{
Parity anomaly of bound states and optical properties in semiconductor superlattices with structural defects
}

\author{
Xue-Hua Wang* \\ CCAST (World Laboratory), P.O. Box 8730, Beijing 100080, China \\ and Institute of Physics, Academia Sinica, P.O. Box 603, Beijing 100080, China \\ Ben-Yuan Gu \\ CCAST (World Laboratory), P.O. Box 8730, Beijing 100080, China; \\ Institute of Physics, Academia Sinica, P.O. Box 603, Beijing 100080, China; \\ and Department of Physics, The University of Hong Kong, Pokfulam Road, Hong Kong, China \\ Guo-Zhen Yang \\ Institute of Physics, Academia Sinica, P.O. Box 603, Beijing 100080, China \\ Jian Wang \\ Department of Physics, The University of Hong Kong, Pokfulam Road, Hong Kong, China
}

(Received 20 November 1997; revised manuscript received 25 March 1998)

\begin{abstract}
We investigate the property of the bound states with an infinite number of classical turning points in the semiconductor superlattices (SL's) with the double-barrier and double-well structural defects. The anomalous parity sequence of these bound states violating the conventional parity rule is predicted. A physical mechanism on the origin of the bound states in the structural defect SL's, different from the mechanism addressed in the previous literature, is proposed. Based upon it, the parity anomaly can be interpreted very well. In addition, we also study the property of the optical transition in this system, for instance, the evaluation of the optical transition probability from the bound states to the bound states, from the bound states to the delocalized scattering states, and from the scattering states to other scattering states, in detail. [S0163-1829(98)02432-1]
\end{abstract}

\section{INTRODUCTION}

It is well known that every eigenstate in the quantum systems with space-reverse symmetry has a definite parity, i.e., the parity of the wave function of the stationary states must be either even or odd under the space reverse transform, as long as the eigenstate is nondegenerate. For the bound states with classical turning points in a onedimensional quantum system, according to the oscillation theorem, ${ }^{1}$ the conventional parity rule is active. The parity of the bound states should alternate between even and odd parity in sequence and the parity of the lowest bound state (ground state) must be even. However, in 1929, Von Neumann and Wigner have suggested a new type of bound state embedded in the continuous energy spectrum (continuum), fundamentally violating the normal parity rule. ${ }^{2}$ Based on a constructive method of amplitude modulation of a freeparticle wave function, they indicated that the spatially oscillating attractive potential could lead to the formation of a bound state lying above the potential barriers. More than twenty years ago, some researchers have addressed that there might exist Von Neumann-Wigner bound states in some atomic and molecular systems ${ }^{3,4}$ and semiconductor heterostructures. ${ }^{5,6}$ It has become a well known fact, through their studies, that the oscillation theorem is not applicable to the bound states embedded in the continuum. Although the Von Neumann-Wigner bound states contribute an example of the parity anomaly of the bound states, more knowledge about this effect cannot be further acquired from this kind of bound state because there only exists one bound state for a given Von Neumann-Wigner-type oscillating potential. The parity anomaly of the von Neumann-Wigner bound states can be simply ascribed to the lack of classical turning points.

Recently, the Bragg reflection conditions were applied in the semiconductor superlattices (SL's) to form the so-called Bragg confined states (BCS's) of electrons above the barrier. $^{7}$ Shortly later, the BCS's were observed in experiments. ${ }^{8-10}$ The BCS's are significantly different from the Von Neumann-Wigner bound states. The former lies in the minigaps of the SL's; by contrast, the latter is embedded in the continuum. Many theoretical studies have clearly shown that the BCS's can be regarded as specific structural defect states. ${ }^{11-14}$ Moreover, there exist both the abovebarrier and the below-barrier bound states in the SL's with structural defects. Apparently, the below-barrier structural defect states not only are the bound states with classical turning points but are also isolated from the continuum. One is motivated to further reveal the parity anomaly of the bound states in new quantum systems and its origin. In this work, we present a detailed investigation of the parity of the belowbarrier bound states in the symmetric SL's with structural defects and a physical picture, different from the physical mechanism addressed in the previous literature, for understanding the origin of the parity anomaly of the related bound states. We also give the evaluation of the optical transition probability of the related states (including the bound states and delocalized scattering states) in this system.

This paper is organized as follows. In Sec. II, we give a 


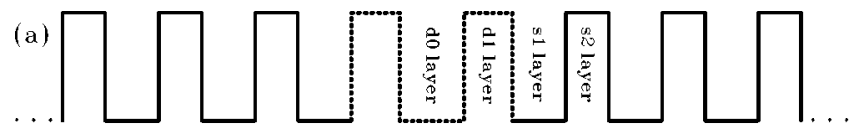

(b)
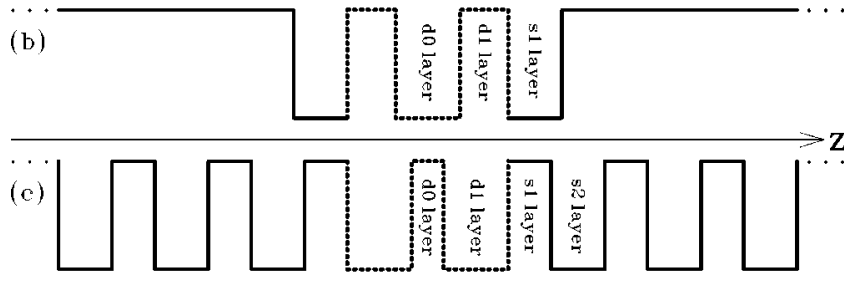

(d)

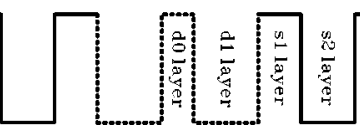

FIG. 1. (a) Schematic diagrams of the SL's with the symmetric double-barrier structure defects (sample No. 1); (b) the corresponding STQWS to sample No. 1; (c) schematic diagrams of the SL's with the symmetric double-well structure defects (sample No. 2); (d) the corresponding SQQWS to sample No. 2. The structural defect regions are plotted with the dotted lines.

brief description of the model structures and present the necessary formulas used in calculations of levels of the bound states. Then, the parity anomaly of the bound states is studied in detail. In Sec. III, we present the expressions of the normalized envelope wave functions for the bound states and the delocalized scattering states. Based upon them, the optical transition characteristics of the system are investigated. Finally, a brief summary is reserved in Sec. IV.

\section{PROPERTIES OF BOUND STATES}

\section{A. Model and calculation of bound levels}

We consider two typical samples of the symmetric SL's with structural defects: (i) symmetric double-barrier structure defect SL's (sample No. 1) in which two identical semiinfinite SL's are coupled by a "distorted" double-barrier structure with different structural unit from the semi-infinite SL's, as shown in Fig. 1(a); (ii) symmetric double-well structure defect SL's (sample No. 2) in which a "distorted", double-well structure is embedded in a SL, as shown in Fig. 1(c). We also draw the schematic diagrams of the related triple- and quadruple-quantum-well structures in order to discuss the origin of the structural defect states, as shown in Figs. 1(b) and 1(d). We choose the growth direction of the SL's as the $Z$ axis and the center of the defect structure as the coordinate origin. For the half-space of $Z>0$, the longitudinal envelope wave function of an electron can be expressed as

$$
\Phi_{d j}(Z)=A_{d j} e^{i k_{d j}\left(Z-Z_{d j}\right)}+B_{d j} e^{-i k_{d j}\left(Z-Z_{d j}\right)}
$$

for the $d j$ th $(d j=d 0, d 1)$ layer in the defect region, and

$$
\Phi_{s j}^{(m)}(Z)=\left[A_{s j} e^{i k_{s j}\left(Z-Z_{s j}^{m}\right)}+B_{s j} e^{-i k_{s j}\left(Z-Z_{s j}^{m}\right)}\right] e^{i k_{z}(m-1) L}
$$

for the $s j$ th $(s j=s 1, s 2)$ layer in the $m$ th period of the right semi-infinite SL. Here $Z_{d j}$ and $Z_{s j}^{m}$ designate the center coordinates of the corresponding layers, $k_{z}$ is the Bloch wave vector, $L$ the period of the semi-infinite SL, and the longitudinal wave number of the electron, $k_{\mu j}(\mu=d, s)$, in the $\mu j$ th layer is given by

$$
k_{\mu j}=\left[\frac{2 m_{\mu j}\left(E-U_{\mu j}\right)}{\hbar^{2}}-k_{x y}^{2}\right]^{1 / 2} .
$$

In Eq. (3), $k_{x y}$ is the transverse wave number of the electron, $E$ the total energy of the electron; $U_{\mu j}=0$ (in the well material) or $U_{0}$ (in the barrier material). $U_{0}$ is the conductionband edge offset, and $m_{\mu j}$ is the energy-dependent effective mass of the electron in the $\mu j$ th layer, incorporating the effect of the band nonparabolicity, it is given by ${ }^{15}$

$$
m_{\mu j}=m_{\mu j}^{0}\left[1+\alpha_{\mu j}\left(E-U_{\mu j}\right)\right],
$$

where $m_{\mu j}^{0}\left(=m_{w}^{0}\right.$ or $\left.m_{b}^{0}\right)$ is the electron effective mass at the conduction-band edge, and $\alpha_{\mu j}\left(=\alpha_{w}\right.$ or $\left.\alpha_{b}\right)$ the nonparabolicity parameter of the band (in the well or barrier material). In terms of Ref. 15, we can infer the relationship between $\alpha_{w}$ and $\alpha_{b}$ as

$$
\alpha_{b}=\frac{m_{w}^{0}}{m_{b}^{0}} \alpha_{w} .
$$

For the structural defect states lying in the minigaps of the $\mathrm{SL}$, the Bloch wave number $k_{z}$ should take a complex value in the form as ${ }^{16}$

$$
k_{z}=\frac{n \pi}{L}+i q \quad(q>0, n=0,1,2, \ldots) .
$$

By using the Bastard boundary conditions at the interfaces of $Z=w_{d 0} / 2, w_{d 0} / 2+w_{d 1}, w_{d 0} / 2+w_{d 1}+w_{s 1}$, and $w_{d 0} / 2+w_{d 1}$ $+w_{s 1}+w_{s 2}\left(w_{\mu j}\right.$ is the width of the $\mu j$ th layer), we can derive the following equations

$$
\begin{gathered}
\left(\begin{array}{c}
A_{d 0} \\
B_{d 0}
\end{array}\right)=\hat{Q}\left(\begin{array}{c}
A_{s 1} \\
B_{s 1}
\end{array}\right), \\
{\left[\hat{I}-(-1)^{n} e^{-q L} \hat{P}\right]\left(\begin{array}{c}
A_{s 1} \\
B_{s 1}
\end{array}\right)=0,}
\end{gathered}
$$

where $\hat{I}$ is a unit matrix. The matrices $\hat{Q}$ and $\hat{P}$ are defined by

$\hat{Q}=\hat{T}^{-1}\left(m_{d 0}, k_{d 0}, w_{d 0}\right) \hat{M}\left(m_{d 1}, k_{d 1}, w_{d 1}\right) \hat{T}\left(m_{s 1}, k_{s 1},-w_{s 1}\right)$,

$\hat{P}=\hat{T}^{-1}\left(m_{s 1}, k_{s 1}, w_{s 1}\right) \hat{M}\left(m_{s 2}, k_{s 2}, w_{s 2}\right) \hat{T}\left(m_{s 1}, k_{s 1},-w_{s 1}\right)$,

with

$$
\begin{gathered}
\hat{M}(m, k, x)=\hat{T}(m, k,-x) \hat{T}^{-1}(m, k, x), \\
\hat{T}(m, k, x)=\left(\begin{array}{cc}
e^{i k x / 2} & e^{-i k x / 2} \\
(i k / m) e^{i k x / 2} & -(i k / m) e^{-i k x / 2}
\end{array}\right) .
\end{gathered}
$$

It is evident from Eq. (1) that the even and odd parity bound states correspond to the conditions $A_{d 0}=B_{d 0}$ and 


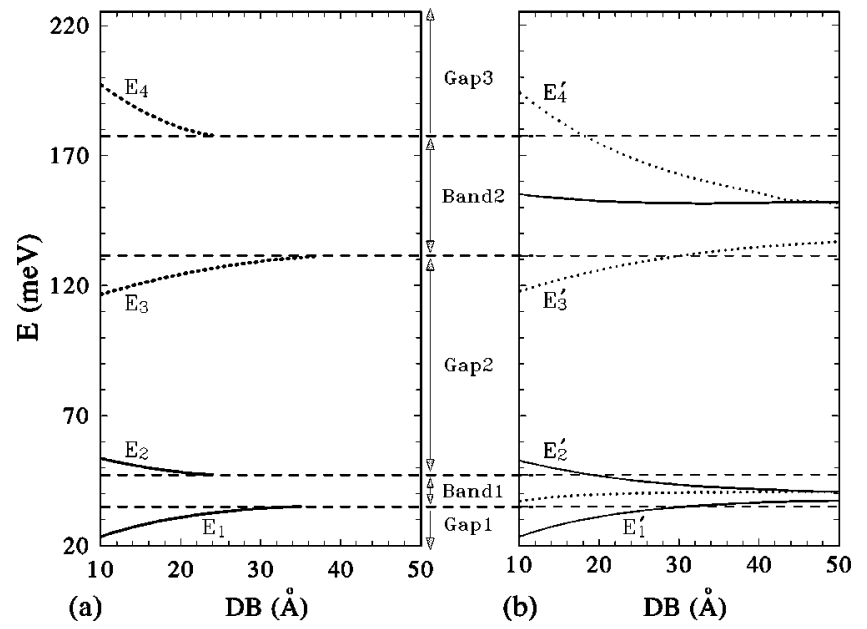

FIG. 2. (a) Dependence of the below-barrier structural defect states on the width $D B$ of two identical defect barriers in sample No. 1; (b) variations of the bound levels with $D B$ in the corresponding STQWS [as shown in Fig. 1(b)] to sample No. 2. Solid (dotted) curves correspond to the even (odd) parity states. Four horizontal dashed lines divide the below-barrier energy spectrum into two minibands and three minigaps.

$A_{d 0}=-B_{d 0}$, respectively. From Eqs. (7) and (8), we deduce the equations for determining the structural defect states as

$$
\begin{aligned}
& \cosh (q L)=0.5(-1)^{n}\left(\hat{P}_{11}+\hat{P}_{22}\right), \\
& {\left[1 \mp(-1)^{n} e^{-q L} \hat{P}_{22}\right]\left(\hat{Q}_{11}-\hat{Q}_{21}\right) \mp(-1)^{n} e^{-q L} \hat{P}_{21}} \\
& \times\left(\hat{Q}_{22} \mp \hat{Q}_{12}\right)=0,
\end{aligned}
$$

where the signs" " $\mp$," correspond to the even and odd parity states, respectively.

\section{B. Parity anomaly of bound states}

We now employ Eqs. (13) and (14) to perform the numerical calculations for both Samples No. 1 and No. 2 composed of $\mathrm{GaAs} / \mathrm{Al}_{0.3} \mathrm{Ga}_{0.7} \mathrm{As}$ material. The well and barrier widths of the semi-infinite SL in two samples are fixed at 80 and $30 \AA$, respectively. The related physical parameters for two samples can be evaluated as follows: ${ }^{17} U_{0}=225 \mathrm{meV}$, $m_{w}^{0}=0.067 m_{e}$, and $m_{b}^{0}=0.092 m_{e}$, where $m_{e}$ is the mass of the free electron. The nonparabolicity parameter $\alpha_{w}$ is taken to be $0.642 \mathrm{eV}^{-1}{ }^{18}$ The first and second allowed minibands of SL expand over the energy ranges of [35.03, 47.20](meV) and $[131.46,177.38](\mathrm{meV})$, respectively. For simplicity, all the calculations were limited to the case of $k_{x y}=0$.

The dependence of the bound-state levels $E_{i}$ on the width $D B\left(=w_{d 1}\right)$ of two identical defect barriers in sample No. 1 [as shown in Fig. 1(a)] is displayed in Fig. 2(a), fixed the defect well width at $85 \AA$. Solid (dotted) curves correspond to the even (odd) parity states. It is clearly seen that the level intervals $\Delta E_{21}=E_{2}-E_{1}$ and $\Delta E_{43}=E_{4}-E_{3}$ of the bound states gradually reduce as $D B$ increases. This result seems to be similar to the level splitting effect in a coupled-doublequantum-well structure when the width of the coupling barrier is broadened. However, the fact is more interesting that these bound states exhibit anomalous parity behavior, substantially violating the conventional parity rule of the bound states. For instance, when $D B<24 \AA$, the parity of both the ground state $E_{1}$ and the first excited state $E_{2}$ is even, whereas the second and the third excited states $\left(E_{3}\right.$ and $\left.E_{4}\right)$ have the odd parity. For $24 \leqslant D B<35 \AA$, only one even parity state $E_{1}$ and one odd parity state $E_{3}$ survive. Their parity sequence accords with the conventional parity rule. When $35 \leqslant D B<38 \AA$, only one odd parity state $E_{3}$ (as the lowest bound state) is survived. These results evidently show that the conventional parity rule is no longer applicable to the bound states in the SL's with the structural defects.

In the previous literature, ${ }^{14}$ it has been addressed that the bound states in the structural defect SL stem from the resonant states or bound states in the separate defect region from the SL. Apparently, the existence of four below-barrier defect bound states in sample No. 1 does not match this physical picture in which only two below-barrier resonant states survive for an isolated double-barrier-defect structure with a defect well of $85 \AA$ width. So, we have to search a new physical mechanism for understanding the parity anomaly of the structural defect states in sample No. 1.

As is well known, besides the bound states, there are the scattering states lying within the minibands in the structural defect SL's. ${ }^{14}$ It is worthy to pointing out that these scattering states do not posses definite parity owing to their double degeneracy. The formation of the minibands is attributed to the splitting of levels due to the periodicity coupling between the adjacent quantum wells in the perfect SL's. When introducing the structural defects into the ideal SL, this periodicity coupling is locally broken down around the structural defect layers. As a result, the periodicity broken coupling between the adjacent quantum wells occurs within the region including the structural defects and two nearest quantum wells to them in the left and right semi-infinite SL's. This periodicity-broken coupling leads to the appearance of new splitting levels, different from the splitting levels stemmed from the periodicity coupling. Some of them may lie within the minibands of the SL's and turn into the delocalized scattering states without definite parity. On the other hand, the other part of them resides in the minigaps of the SL's and becomes the bound states. Therefore, one may reasonably conjecture that the below-barrier structural defect states in sample No. 1 originate from some of the bound states in the related symmetric-triple-quantum-well structure (STQWS) as shown in Fig. 1(b). To confirm this conjecture, we examine the dependence of the bound levels on the width $D B$ of two identical barriers in the STQWS. The results are shown in Fig. 2(b). It can be seen that the first excited state (no marked dotted line between $E_{1}^{\prime}$ and $E_{2}^{\prime}$ states) with odd parity and the fourth excited state (no marked solid line between $E_{3}^{\prime}$ and $E_{4}^{\prime}$ states) with even parity just respectively fall within the first and second minibands of the SL when 10 $\leqslant D B \leqslant 50 \AA$. Consequently, when the left and right semiinfinite barrier regions in the STQWS are replaced by two semi-infinite SL's, these two bound states tend to turn into the delocalized scattering states without definite parity as they merge with the minibands of the SL. On the other hand, it is noted that the structural defect states $E_{i}$ in sample No. 1 and its analogous ones $E_{i}^{\prime}$ in the STQWS exhibit quite simi- 


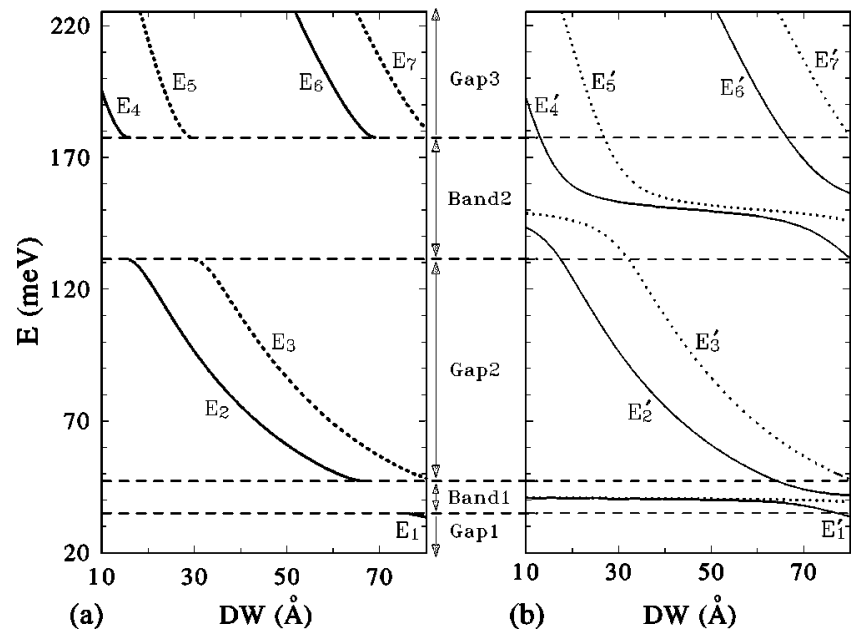

FIG. 3. (a) Dependence of the below-barrier structural defect states on the width $D W$ of two identical defect wells in sample No. 2; (b) variations of the bound levels with $D B$ in the corresponding SQQWS [as shown in Fig. 1(d)] to sample No. 2. Solid (dotted) curves correspond to the even (odd) parity states. Four horizontal dashed lines divide the below-barrier energy spectrum into two minibands and three minigaps.

lar varying tendency. Moreover, the $D B$ value for $E_{i}$ state disappearing at the miniband edge is very close to the one for $E_{i}^{\prime}$ state being truncated by the miniband edge. Hence, it is believed with certainty that the structural defect states $E_{i}$ in sample No. 1 are the development of the bound states $E_{i}^{\prime}$ when the semi-infinite barrier regions in the STQWS are replaced by two semi-infinite SL's. The parity anomalous sequence of the bound states in the sample No. 1 arises from the fact that during this evolution two of the abovementioned bound states in the STQWS turn into the delocalized scattering states without definite parity.

To further support the above-mentioned conclusion we present another evidence by envisaging sample No. 2 [as shown Fig. 1(c)] with the fixed defect barrier width of $20 \AA$. The variation of the bound levels with the width $D W\left(D W=w_{d 1}\right)$ of two identical defect wells in sample No. 2 is illustrated in Fig. 3(a). The even (odd) parity state levels are plotted with solid (dotted) lines. It is evident that all the structural defect state levels $E_{i}$ monotonically decrease and lastly merge into the minibands as the $D W$ increases. When broadening $D W$ from 10 up to $66 \AA$, the number of below-barrier bound states increase from 1 up to 4. The parity of these bound states obeys the conventional parity rule. However, when $66<D W \leqslant 69 \AA$, there are three bound states, i.e., $E_{3}, E_{6}$, and $E_{7}$. Their parity is in turn odd, even, and odd. When $69<D W \leqslant 75 \AA$, only two odd parity states are survived owing to the disappearance of $E_{6}$ state. When $75<D W \leqslant 80 \AA$, we can find three bound states due to the emergence of $E_{1}$ state, and their parity is in turn even, odd, and odd. Apparently, when $66<D W \leqslant 80 \AA$, the parity of the bound states in sample No. 2 completely violates the conventional parity rule. To get better insight into the origin of the parity anomaly, we examine the behaviors of the bound states in the related symmetric quadruple-quantumwell structure (SQQWS) as shown in Fig. 1(d). The dependence of these bound levels on $D W$ is displayed in Fig. 3(b). It is noted that the first excited state with the odd parity (between $E_{1}^{\prime}$ and $E_{2}^{\prime}$ states) completely falls within the first miniband of the SL. It tends to turn into a delocalized scattering state without definite parity when the semi-infinite barrier regions in the SQQWS are replaced by two semiinfinite SL's. Comparing Figs. 3(a) with 3(b), we can find the same similarities between the defect bound state $E_{i}$ and its analogous one $E_{i}^{\prime}$ as that seen in Figs. 2(a) and 2(b). This brings us a suggestion once again that the bound state $E_{i}$ in sample No. 2 doubtless is the development of the corresponding state $E_{i}^{\prime}$ when the semi-infinite barrier regions in the SQQWS are replaced by two identical semi-infinite SL's. It is worth pointing out that during this evolution as soon as the related $E_{i}^{\prime}$ state are merged into the minibands, the corresponding $E_{i}$ state to $E_{i}^{\prime}$ state disappears from the bound state series. It is this reason that leads to the parity anomaly of the survival bound states in sample No. 2 .

\section{OPTICAL TRANSITION CHARACTERISTICS}

Since the famous optical-absorption experiment on the multiwell structures done by Dingle, ${ }^{19}$ the measurements of the optical-transition spectra, such as the absorption and emission spectra, and the photoluminescence and photoluminescence excited spectra, etc., have become a powerful tool to explore the quantum properties in the quantum wells and SL's. The optical-transition spectra in these structures are determined by the transition probabilities between the related electronic states. It can be expected that the existence of the bound states and their anomalous parity sequence in the structural defect SL's leads to some new optical-transition characteristics, different from that in the complete SL's. We are now in a position to investigate the optical transition characteristics in the SL's with structural defects. For simplicity, we ignore the dependence of the sublevels and minibands on the transverse wave number $k_{x y}$. So, the optical transition probability from an initial state $E_{i}$ to a final state $E_{f}$ in the approximation of an isotropic conduction band can be expressed as

$$
W_{i f}=\frac{2 \pi}{\hbar}\left(\frac{e A_{0}}{m^{*}}\right)^{2}\left|\left\langle\Phi_{f}(Z)\left|\hat{p}_{z}\right| \Phi_{i}(Z)\right\rangle\right|^{2} \delta\left(E_{f}-E_{i}-\hbar \omega\right),
$$

where the function $\delta\left(E_{f}-E_{i}-\hbar \omega\right)$ reflects the conservation of energy; $m^{*}$ is the effective mass of the electron; $A_{0}$ and $\omega$ are respectively the amplitude and frequency of the optical excitation wave; $\Phi_{i, f}(Z)$ are the envelope wave function of the electron along the $Z$ direction. In order to calculate the transition probability $W_{i f}$, we have to take into account the normalization of the related envelope wave functions.

\section{A. Normalization of envelope wave functions for bound and delocalized states}

First, we discuss the normalization of the envelope wavefunction of the bound states. We introduce the relative coordinate in each layer for the half-space of $Z \geqslant 0$ as follows:

$$
\begin{gathered}
\widetilde{Z}_{d 0}=Z-Z_{d 0} \quad\left(0 \leqslant \widetilde{Z}_{d 0} \leqslant w_{d 0} / 2\right), \\
\widetilde{Z}_{d 1}=Z-Z_{d 1} \quad\left(-w_{d 1} / 2 \leqslant \widetilde{Z}_{d 1} \leqslant w_{d 1} / 2\right),
\end{gathered}
$$




$$
\widetilde{Z}_{s j}^{m}=Z-Z_{s j}^{m} \quad\left(-w_{s j} / 2 \leqslant \widetilde{Z}_{s j}^{m} \leqslant w_{s j} / 2\right) .
$$

So, according to Eqs. (1), (2), and (6), the envelope wave function of the bound states for $Z \geqslant 0$ can be reexpressed as

$$
\Phi_{E_{ \pm}}(Z)=\left\{\begin{array}{l}
A_{d 0}^{ \pm} F\left(1, \pm 1, k_{d 0}, \widetilde{Z}_{d 0}\right), \\
A_{d 0}^{ \pm} F\left(A_{d 1}^{ \pm}, B_{d 1}^{ \pm}, k_{d 1}, \widetilde{Z}_{d 1}\right), \\
A_{d 0}^{ \pm}\left[(-1)^{n} e^{-q_{ \pm} L}\right]^{m-1} F\left(A_{s j}^{ \pm}, B_{s j}^{ \pm}, k_{s j}, \widetilde{Z}_{s j}^{m}\right),
\end{array}\right.
$$

where $A_{d 0}$ is the normalized constant; the signs " \pm ", correspond to even and odd parity states; $E_{ \pm}$and $q_{ \pm}$are determined by Eqs. (13) and (14). The function $F(A, B, k, Z)$ is defined as

$$
F(A, B, k, Z)=A e^{i k Z}+B e^{-i k Z} .
$$

By using Bastard boundary conditions at interfaces, we obtain

$$
\begin{aligned}
\left(\begin{array}{c}
A_{d 1}^{ \pm} \\
B_{d 1}^{ \pm}
\end{array}\right) & =\hat{S}_{d 1, d 0}\left(\begin{array}{c}
1 \\
\pm 1
\end{array}\right), \\
\left(\begin{array}{c}
A_{s 1}^{ \pm} \\
B_{s 1}^{ \pm}
\end{array}\right) & =\hat{S}_{s 1, d 1}\left(\begin{array}{c}
A_{d 1}^{ \pm} \\
B_{d 1}^{ \pm}
\end{array}\right), \\
\left(\begin{array}{c}
A_{s 2}^{ \pm} \\
B_{s 2}^{ \pm}
\end{array}\right) & =\hat{S}_{s 2, s 1}\left(\begin{array}{c}
A_{s 1}^{ \pm} \\
B_{s 1}^{ \pm}
\end{array}\right),
\end{aligned}
$$

with

$$
\hat{S}_{\mu, \nu}=\hat{T}^{-1}\left(m_{\mu}, k_{\mu},-w_{\mu}\right) \hat{T}\left(m_{\nu}, k_{\nu}, w_{\nu}\right) .
$$

In terms of the normalized condition of the bound-state wave functions with a definite parity $\int_{0}^{\infty}\left|\Phi_{E_{ \pm}}(Z)\right|^{2}=1 / 2$, we have

$$
A_{d 0}^{ \pm}=\frac{1}{\sqrt{2\left(I_{d}^{ \pm}+I_{s l}^{ \pm}\right)}}
$$

with

$$
\begin{aligned}
I_{d}^{ \pm}= & \int_{0}^{w_{d 0} / 2}\left|F\left(1, \pm 1, k_{d 0}, Z\right)\right|^{2} d Z \\
& +\int_{-w_{d 1} / 2}^{w_{d 1} / 2}\left|F\left(A_{d 1}^{ \pm}, B_{d 1}^{ \pm}, k_{d 1}, Z\right)\right|^{2} d Z,
\end{aligned}
$$

and

$$
I_{s l}^{ \pm}=\frac{1}{1-e^{-2 q_{ \pm} L}} \sum_{i=1}^{2} \int_{-w_{s i} / 2}^{w_{s i} / 2}\left|F\left(A_{s i}^{ \pm}, B_{s i}^{ \pm}, k_{s i}, Z\right)\right|^{2} d Z
$$

We now consider the normalization of the wave functions of the delocalized states with double degeneracy. The energy dispersion relation $E\left(k_{z}\right)$ of the scattering states is determined by

$$
\cos \left(k_{z} L\right)=\frac{1}{2}\left(\hat{P}_{11}+\hat{P}_{22}\right)=\frac{1}{2}\left(\hat{P}_{11}^{\prime}+\hat{P}_{22}^{\prime}\right),
$$

where the matrix $\hat{P}$ is given in Eq. (10) and the matrix $\hat{P}^{\prime}$ is defined as

$$
\hat{P}^{\prime}=\hat{T}\left(m_{s 2}, k_{s 2}, w_{s 2}\right) \hat{M}\left(m_{s 1}, k_{s 1}, w_{s 1}\right) \hat{T}\left(m_{s 2}, k_{s 2},-w_{s 2}\right) .
$$

Out of the defect region, the wave functions of the delocalized scattering states should be the superposition of $k_{z}$ and $-k_{z}$ states Bloch wave functions. Considering the structural symmetry, the two linearly independent wave functions (for the half space of $Z \geqslant 0$ ) of the delocalized scattering states with an energy $E\left(k_{z}\right)=E\left(-k_{z}\right)$ lying within the minibands can be expressed in terms of the even and odd parity states as

$$
\Phi_{k_{z}}^{ \pm}(Z)=\left\{\begin{array}{l}
C_{d 0}^{ \pm} F\left(1, \pm 1, k_{d 0}, \widetilde{Z}_{d 0}\right), \\
C_{d 0}^{ \pm} F\left(C_{d 1}^{ \pm}, D_{d 1}^{ \pm}, k_{d 1}, \widetilde{Z}_{d 1}\right), \\
C_{d 0}^{ \pm}\left[C_{s j}^{ \pm} G_{s j}\left(k_{z}, \widetilde{Z}_{s j}^{m}\right)+D_{s j}^{ \pm} G_{s j}\left(-k_{z}, \widetilde{Z}_{s j}^{m}\right)\right],
\end{array}\right.
$$

where $C_{d 0}^{ \pm}$is a normalization constant. The function $G_{s j}\left(k_{z}, \widetilde{Z}_{s j}^{m}\right)$ is defined as

$$
G_{s j}\left(k_{z}, \widetilde{Z}_{s j}^{m}\right)=F\left(1, \Theta_{s j}\left(k_{z}\right), k_{s j}, \widetilde{Z}_{s j}^{m}\right) e^{i k_{z}(m-1) L}
$$

with

$$
\Theta_{s 1}\left(k_{z}\right)=\frac{\hat{P}_{21} e^{i k_{z} L}}{1-\hat{P}_{22} e^{i k_{z} L}}
$$

and

$$
\Theta_{s 2}\left(k_{z}\right)=\frac{\hat{P}_{21}^{\prime} e^{i k_{z} L}}{1-\hat{P}_{22}^{\prime} e^{i k_{z} L}} .
$$

Applying the Bastard boundary conditions at interfaces to the wave functions $\Phi_{k_{z}}^{ \pm}\left(k_{z}, Z\right)$, we can obtain the equations for determining the coefficients $\left\{\begin{array}{l}C_{d 1}^{ \pm} \\ D_{d 1}^{ \pm}\end{array}\right\}$and $\left\{\begin{array}{l}C_{s j}^{ \pm} \\ D_{s j}^{ \pm}\end{array}\right\}$as follows:

$$
\begin{gathered}
\left(\begin{array}{c}
C_{d 1}^{ \pm} \\
D_{d 1}^{ \pm}
\end{array}\right)=\hat{S}_{d 1, d 0}\left(\begin{array}{c}
1 \\
\pm 1
\end{array}\right), \\
\left(\begin{array}{c}
C_{s 1}^{ \pm} \\
D_{s 1}^{ \pm}
\end{array}\right)=\hat{R}_{s 1}^{-1} \hat{S}_{s 1, d 1}\left(\begin{array}{c}
C_{d 1}^{ \pm} \\
D_{d 1}^{ \pm}
\end{array}\right), \\
\left(\begin{array}{c}
C_{s 2}^{ \pm} \\
D_{s 2}^{ \pm}
\end{array}\right)=\hat{R}_{s 2}^{-1} \hat{S}_{s 2, s 1} \hat{R}_{s 1}\left(\begin{array}{l}
C_{s 1}^{ \pm} \\
D_{s 1}^{ \pm}
\end{array}\right),
\end{gathered}
$$

where the matrix $\hat{R}_{s j}$ is defined as

$$
\hat{R}_{s j}=\left(\begin{array}{cc}
1 & 1 \\
\Theta_{s j}\left(k_{z}\right) & \Theta_{s j}\left(-k_{z}\right)
\end{array}\right) .
$$

The wave function in the continuum (miniband) spectrum can be normalized according to

$$
\int_{-\infty}^{+\infty} \Phi_{k_{z}}^{ \pm}(Z)\left[\Phi_{k_{z}^{\prime}}^{ \pm}(Z)\right]^{*} d Z=\delta\left(k_{z}-k_{z}^{\prime}\right) .
$$


After considering the definite parity of wave functions, the normalization condition can be rewritten as

$$
\begin{gathered}
2 \int_{0}^{w_{d 0} / 2+w_{d 1}} \Phi_{k_{z}}^{ \pm}(Z)\left[\Phi_{k_{z}^{\prime}}^{ \pm}(Z)\right]^{*} d Z+2 \int_{w_{d 0} / 2+w_{d 1}}^{+\infty} \Phi_{k_{z}}^{ \pm}(Z) \\
\times\left[\Phi_{k_{z}^{\prime}}^{ \pm}(Z)\right]^{*} d Z=\delta\left(k_{z}-k_{z}^{\prime}\right) .
\end{gathered}
$$

The first term in Eq. (38) has a finite value, and its contribution can be neglected with respect to the second term. By using the relation

$$
\lim _{N \rightarrow \infty} \sum_{m=1}^{N} e^{ \pm i\left(k_{z}-k_{z}^{\prime}\right)(m-1) L}=\delta\left(k_{z}-k_{z}^{\prime}\right),
$$

we can obtain the normalized coefficient

$$
C_{d 0}^{ \pm}=\frac{1}{\sqrt{2 I^{ \pm}}},
$$

where

$$
\begin{aligned}
I^{ \pm}= & \sum_{i=1}^{2}\left[\left|C_{s i}^{ \pm}\right|^{2} \int_{-w_{s i} / 2}^{w_{s i} / 2}\left|F\left(1, \Theta_{s i}\left(k_{z}\right), k_{s i}, Z\right)\right|^{2} d Z\right. \\
& \left.+\left|D_{s i}^{ \pm}\right|^{2} \int_{-w_{s i} / 2}^{w_{s i} / 2}\left|F\left(1, \Theta_{s i}\left(-k_{z}\right), k_{s i}, Z\right)\right|^{2} d Z\right]
\end{aligned}
$$

\section{B. Numerical results and discussions}

Substituting the normalized wave functions given in Eqs. (19) and (30) into Eq. (15), we can easily calculate the optical transition probability. As an example, we perform the numerical calculation for the double-barrier structure defect SL [as shown in Fig. 1(a)] with the barrier width of $15 \AA$. Because the dipole transition between two bound states with identical parity is forbidden, thus the transition probability between the $E_{1}$ and $E_{2}$ states vanishes, and so does the transition probability between the $E_{3}$ and $E_{4}$ states. The permitted transition probabilities between two bound states are respectively $W_{13}=0.2369 \times 10^{-5}\left(C_{0}\right), \quad W_{14}=0.1339$ $\times 10^{-3}\left(C_{0}\right), \quad W_{23}=0.7717 \times 10^{-3}\left(C_{0}\right)$, and $W_{24}=0.1172$ $\times 10^{-4}\left(C_{0}\right)$, here the unit $C_{0}$ is defined as

$$
C_{0}=2 \pi \hbar\left(\frac{e A_{0}}{m^{*}}\right)^{2} \text {. }
$$

These numerical results show that the transition probabilities from $E_{1}$ to $E_{4}$ states and from $E_{2}$ to $E_{3}$ states are the same order of magnitude, but they are approximately one or two orders of magnitude larger than the probability from $E_{1}$ to $E_{3}$ states or from $E_{2}$ to $E_{4}$ states. Note that $E_{1}$ and $E_{4}$ states lie within the first and third minigaps, and the values of their corresponding index $n$ [given by in Eq. (6)] are an even number ( $n=0$ and 2), however, both the $E_{2}$ and $E_{3}$ states are located at the second minigap, and the values of their corresponding index $n$ are odd number ( $n=1$ and 3 ). That is to say, the transition probability between two bound states with identical odd/even property of their index $n$ is larger than that between two bound states with different odd/even property of their index $n$.
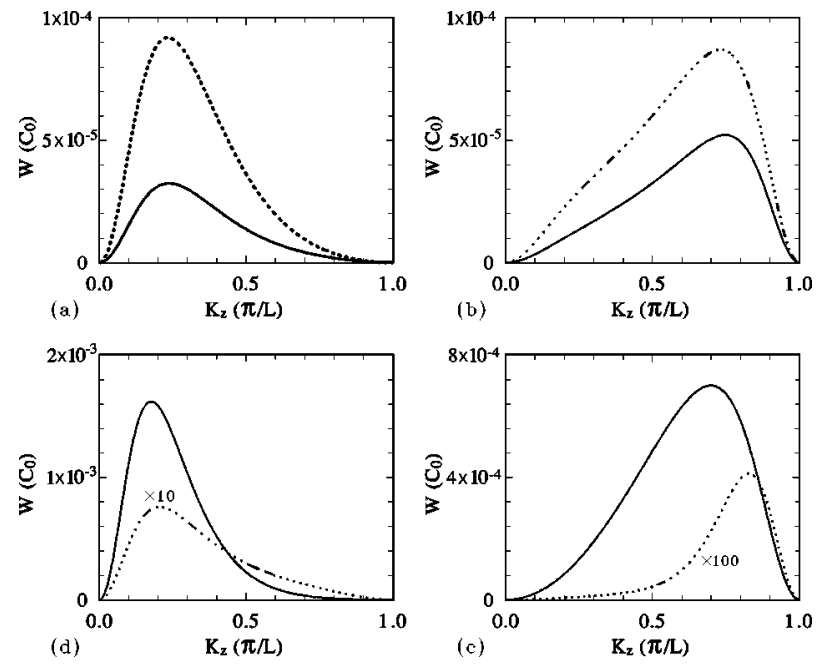

FIG. 4. Transition probabilities from the bound states to the delocalized states in the double-barrier structure defect SL stated in the text. (a), (b), (c), and (d) correspond to the transitions from the levels $E_{1}, E_{2}, E_{3}$, and $E_{4}$ to two minibands, respectively. The dotted and the solid lines represent the transition probabilities from the levels $E_{i}$ to the first and second minibands, respectively.

The transition characteristics among the bound states and scattering states are displayed in Figs. 4(a)-4(d). Figures 4(a), 4(b), 4(c), and 4(d) demonstrate the variation of the transition probability from the $E_{1}, E_{2}, E_{3}$, and $E_{4}$ levels to two minibands of the SL with the Bloch wave number $k_{z}$. Dotted (solid) lines correspond to the transitions from the bound levels to the first (second) miniband. From Figs. 4(a)4(d), it is evident that the transition spectra from the bound states to the delocalized states exhibit the interesting features: (i) The transition probabilities from the bound states to the delocalized states located at the center and edge of the Brillouin zone are quite small; (ii) there always exists a maximum in every transition probability plot; (iii) the positions of these maxima depend on the odd/even property of the index $n$ of the bound states. As the index $n$ of the bound states is even number, the peak position closes the center of the Brillouin zone, otherwise the peak appears to be close to the edge of the Brillouin zone; (iv) the transition probabilities from the bound states with even parity to two minibands

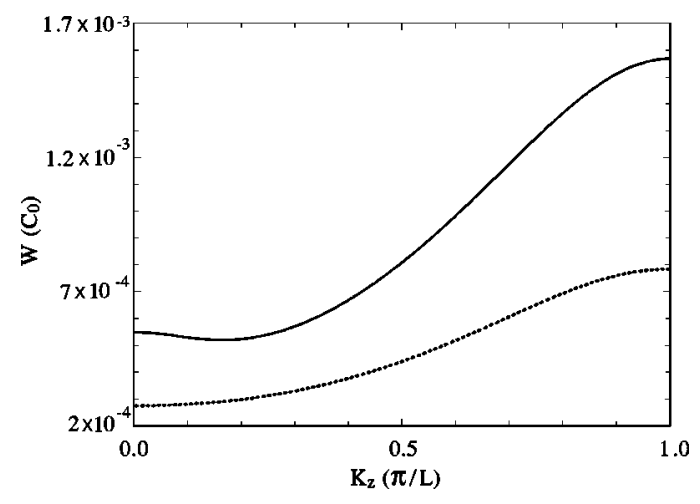

FIG. 5. Transition probabilities between two minibands for the same double-barrier structure defect SL as in Fig. 4 and for the corresponding complete SL. The solid and dotted lines correspond to the cases of the defect SL and complete SL, respectively. 
are of the same order of magnitude, while the ones from the bound states with odd parity to two minibands possess quite different order of magnitude.

The transition probability between two minibands as a function of the Bloch wave number $k_{z}$ of the SL for the double-barrier structure defect SL and the corresponding complete SL is displayed in Fig. 5. The solid line and dotted line correspond to the defect SL and the complete SL. Comparing the solid line and the dotted line, it is found that except for the values in the defect SL being larger than those in the complete SL, they both exhibit a similarly varying tendency: the transition probability in the whole Brillouin zone possesses the same order of magnitude, which is completely different from the transition from the bound states to the delocalized states; and the transition probability between two delocalized states at the edge of the Brillouin zone is larger than that at the center of the Brillouin zone.

All of the above optical-transition characteristics in the structural defect SI may be measured by the infrared absorption and emission spectra. Apparently, the optical absorption between the $E_{1}$ and $E_{2}$ states and the optical emission between the $E_{4}$ and $E_{3}$ states cannot be observed in the infrared absorption and emission spectra. It arises from the reason that the optical transition between two bound states with identical parity is forbidden. So, we suggest that the anomalous parity sequence in the structural defect SL's can be identified by measurements of the related infrared absorption and emission spectra.

\section{SUMMARY}

We have presented a detailed analyses on the properties of the below-barrier defect states with an infinite number of classical turning points in the semiconductor SL's with the structural defects. The anomalous parity sequence of the structural defect states is predicted. This parity anomaly arises from the following source: The structural defect states stem from splitting of levels caused by the periodicitybroken coupling between the adjacent quantum wells in the region composed of the structural defects and two nearest quantum wells to them in the left and right semi-infinite SL's. The parity sequence of these splitting levels obeys the conventional parity rule. When some of these splitting levels are merged into the minibands of the SL's and become the delocalized scattering states without definite parity, the parity sequence of the final survival structural defect states exhibits anomalous. In addition, we evaluate various transition probabilities, for instance, from the bound states to the bound states, from the bound states to the delocalized states, and from the delocalized states to other delocalized states in the structural defect SL. It is found that the transition probability strongly depends on the properties of the states involving the transition process, for instance, their localization status, the state parity, and the odd/even parity of the index $n$ of the bound states. Finally, we suggest that the anomalous parity sequence in the structural defect SL's can be identified by measurements of the related infrared absorption and emission spectra.

\section{ACKNOWLEDGMENTS}

We gratefully acknowledge financial support through a research grant from the Chinese National Science Foundation, a research grant from the Croucher Foundation, a RGC grant from the SAR Government of Hong Kong under Grant No. HKU 261/95P, and a CRCG grant from the University of Hong Kong.
*Electronic address: wangxh@aphy01.iphy.ac.cn

${ }^{1}$ L. D. Landau and E. M. Lifshitz, Quantum Mechanics, Nonrelativistic Theory, translated by J. B. Sykes and J. S. Bell (Pergamon, London, 1958), p. 58.

${ }^{2}$ J. Von Neumann and E. Wigner, Phys. Z. 30, 465 (1929).

${ }^{3}$ F. H. Stillinger and T. A. Weber, Phys. Rev. A 10, 1122 (1974).

${ }^{4}$ F. H. Stillinger and D. R. Herrick, Phys. Rev. A 11, 446 (1974).

${ }^{5}$ D. R. Herrick, Physica B 85, 44 (1977).

${ }^{6}$ F. H. Stillinger, Physica B 85, 270 (1977).

${ }^{7}$ G. Lenz and J. Salzman, Appl. Phys. Lett. 56, 871 (1990).

${ }^{8}$ F. Capasso, C. Sirtori, J. Faist, D. L. Sivco, S. N. G. Chu, and A. Y. Cho, Nature (London) 358, 565 (1992).

${ }^{9}$ C. Sirtori, F. Capasso, J. Faist, D. L. Sivco, S. N. G. Chu, and A. Y. Cho, Appl. Phys. Lett. 61, 898 (1992).

${ }^{10}$ M. Zahler, I. Brener, G. Lenz, J. Salzman, E. Cohen, and L.
Pfeiffer, Appl. Phys. Lett. 61, 949 (1992).

${ }^{11}$ G. Bastard, Phys. Rev. B 25, 7584 (1982).

${ }^{12}$ R. A. Suris and P. Lavallard, Phys. Rev. B 50, 8875 (1994).

${ }^{13}$ T. A. Weber, Solid State Commun. 90, 713 (1994).

${ }^{14}$ D. Indjin, V. Milanovic, and Z. Ikonic, Phys. Rev. B 52, 16762 (1995); 55, 9722 (1997).

${ }^{15}$ D. F. Nelson, R. C. Miller, and D. A. Kleinman, Phys. Rev. B 35, 7770 (1987).

${ }^{16} \mathrm{H}$. Jones, Theory of Brillouin Zones and Electronic States in Crystals (North-Holland, Amsterdam, 1960).

${ }^{17}$ X. H. Wang, B. Y. Gu, and G. Z. Yang, Phys. Rev. B 55, 9340 (1997).

${ }^{18}$ E. Ekenberg, Phys. Rev. B 40, 7714 (1989).

${ }^{19} \mathrm{R}$. Dingle, in Advance in Solid State Physics (Braumschweig, Vieweg, 1975), Vol. 15, p. 21. 